\title{
Association Between Perceived Demands and Musculoskeletal Disorders Among Hospital Nurses of Shiraz University of Medical Sciences: A Questionnaire Survey
}

\author{
Alireza Choobineh \\ Abdolreza Rajaeefard \\ Masoud Neghab
}

School of Health, Shiraz University of Medical Sciences, Shiraz, Iran

Excessive demands on nurses may result in high rates of musculoskeletal disorders (MSDs). This study was conducted among hospital nurses of Iran's Shiraz University of Medical Sciences (SUMS) to determine the prevalence of MSDs and to examine the relationship between perceived demands and reported MSDs. In this study, 641 randomly selected nurses from 12 SUMS hospitals participated. The Nordic musculoskeletal disorders questionnaire and Job Content Questionnaire were used as collecting data tools.

The results showed that $84.4 \%$ of the participants had experienced some form of symptoms of MSDs during the past 12 months. Lower back symptoms were found to be the most prevalent problem. Perceived physical demands were significantly associated with MSDs $(O R=1.5-2.7)$. No association was found between perceived psychological demands and reported symptoms. It was concluded that any intervention program for preventing MSDs among SUMS hospital nurses had to focus on reducing physical demands, particularly excessive postural demands.

Iran musculoskeletal disorders nurses perceived demands

\section{INTRODUCTION}

Musculoskeletal disorders (MSDs) represent a leading cause of occupational injury and disability in the developed and industrially developing countries $[1,2,3,4,5,6]$. Risk factors are known to include workplace activities such as heavy lifting and repetitive tasks [7], while demographic characteristics and psychosocial factors are also known to be important predictive variables $[8$, $9,10]$. Because of the requirements of a nursing job, hospital nurses form an occupational group regularly affected by MSDs [4, 11, 12, 13, 14]. Epidemiological studies have shown that hospital nursing is a high-risk occupation for developing work-related MSDs [15, 16, 17, 18]. For instance, low back pain/injury, neck and shoulder problems have been reported to be common MSDs among nursing personnel $[12,13,15,16,17,19,20]$. Some studies have shown that overexertion injuries might cause nurses to leave their profession. Owen pointed out that $20 \%$ of nurses with back pain had made employment transfer at least once [21].

Nursing has been recognized as a physically demanding occupation [13, 22]. Concerning physical workload, the nursing profession ranks second after industrial work [23]. Bending, twisting, sudden movement in non-neutral postures and manual handling, in particular, patient handling are common in it.

Funding through Shiraz University of Medical Sciences, contract 83-2219, supported this investigation.

Correspondence and requests for offprints should be sent to Alireza Choobineh, Occupational Health Department, School of Health, Shiraz University of Medical Sciences, Shiraz, Iran. E-mail: <alrchoobin@sums.ac.ir>. 
Given these facts, as a first step toward reducing MSDs and their consequences among Iran's Shiraz University of Medical Sciences (SUMS) hospital nurses, this study was conducted to determine the prevalence of MSDs among SUMS hospital nurses, to identify major factors associated with MSD symptoms in the study population, and to examine the relationship between perceived demands and reported MSDs in different body regions of SUMS hospital nurses.

\section{METHODS}

\subsection{Design and Samples}

In this cross-sectional study conducted between February and September 2005, data were collected via anonymous questionnaires. A random sample of 641 registered, professionally active nurses with at least one-year job tenure was selected from different wards of 12 SUMS hospitals.

The six-page questionnaire consisted of three parts and covered the following items: personal details (weight, height, age, gender, job tenure, daily working time, marital status, number of children, education, health and medical background); MSDs in different body regions; and job demands. Reported MSDs were limited to the past 12 months. All subjects received the questionnaire in person in their workplace. They had $30 \mathrm{~min}$ to complete the questionnaire and return it to the researcher.

\subsection{Measurement of Variables}

The general Nordic questionnaire of musculoskeletal symptoms [24] was used to examine reported cases of MSDs among the study population. The Job Content Questionnaire (JCQ) was used to measure perceived job demands [25]. The JCQ contains items on both physical and psychological aspects of work demands. It has been used to study the relation between work demands and work-related outcomes (i.e., MSDs, cardiovascular disease, etc.) in different countries [26]. Additional occupation-specific physical demand items for the nursing job, as recommended by Trinkoff, Lipscomb, GeigerBrown, et al. [13], were also included. Physical and psychological demands were measured using 12- and 8-item JCQ scales, respectively (see the Appendix for the items). Each item was scored based on a 4-point scale (i.e., strongly agree to strongly disagree or often to never).

Based on Trinkoff et al.'s methodology [13], responses for perceived physical demands were dichotomized (1 and 2 vs. 3 and 4) and summed to produce total scores ranging from 0 to 12 . The results were then categorized as low (0-2), medium (3-9), and high (10-12) physical demands. Items for perceived psychological demands were dichotomized as well (1 and 2 vs. 3 and 4) and summed into a demand score to produce total scores ranging from 0 to 8 . Perceived psychological demands were then categorized as low (0-5) and high (6-8).

In order to estimate the reliability of the responses to questions in the questionnaire, the test-retest method was applied [27] on 5\% of the study population.

\subsection{Data Analysis}

Upon completion of the field survey and data collection, data were transferred into the computer for statistical analysis. Statistical analyses were performed using SPSS version 11 and STATA release 7. Chi-square test was used to assess univariate associations between perceived variables and reported MSDs. Multiple logistic regression analysis was performed for each outcome retaining the perceived variables in the models to adjust for potential confounding.

\section{RESULTS}

Table 1 summarizes personal details of the nurses who participated in the study. Table 2 presents the prevalence of MSD symptoms in different body regions of the nurses during the past 12 months. It is clear that the most commonly affected region among the nurses was the lower back (54.9\%).

The participants' reports revealed that during the past 12 months there were totally 1,520 
TABLE 1. Some Personal Details of the Nurses Who Participated in the Study $(n=641)$

\begin{tabular}{lcc}
\hline Gender (\%) & female/male & $84.7 / 15.3$ \\
Age (years) & $M(S D)$ range & $32.03(8.02) 22-66$ \\
Weight (kg) & $M(S D)$ range & $61.26(10.02) 41.5-100.0$ \\
Height (cm) & $M(S D)$ range & $163.16(7.58) 136-190$ \\
Body Mass Index & $M(S D)$ range & $23.01(3.29) 15.42-39.16$ \\
Job tenure (years) & $M(S D)$ range & $8.71(7.77) 1-30$ \\
Daily working time (hrs) & $M(S D)$ range & $8.3(2.25) 4-18$ \\
Marital status (\%) & single/married & $41.9 / 58.1$ \\
Children (\%) & yes/no & $60.1 / 39.9$ \\
Education (\%) & associate diploma & 4.3 \\
& B.Sc. & 93.2 \\
Second job (\%) & $M . S c$. & 2.5 \\
Working schedule (\%) & yes/no & $9.8 / 90.2$ \\
Overall patient-to-nurse ratio per shift & shifts/fixed & $87.3 / 12.7$ \\
\hline
\end{tabular}

TABLE 2. Frequency of Reported Symptoms in Different Body Regions During the 12 Months Prior to the Study $(n=641)$

\begin{tabular}{lc}
\hline Body Region & $\%$ \\
\hline Neck & 36.4 \\
Shoulders & 39.8 \\
Elbows & 17.9 \\
Wrists/hands & 39.3 \\
Upper back & 46.4 \\
Lower back & 54.9 \\
Thighs & 29.3 \\
Knees & 48.4 \\
Legs/feet & 52.1 \\
\hline
\end{tabular}

days of sick leave due to MSDs. Accordingly, the average sick leave for each nurse was 2.37 days/year $(S D=8.68)$. Due to MSDs, during the preceding year $31 \%$ of the study population had to visit a physician, $18.4 \%$ had to take sick leave and $20 \%$ had to use physiotherapy services. Twenty-one percent of the nurses declared that MSDs had caused them to change their wards and $18.15 \%$ of them had to quit their jobs temporarily because of MSDs. Twenty-one percent of the nurses believed that MSDs would cause them to change their jobs in future.

Table 3 presents significant factors associated with MSDs for each body region, which are the result of a multiple logistic regression analysis performed to adjust for potential confounding. As Table 3 shows, some items of perceived physical demands are significantly associated with reported MSDs of different body regions. Awkward posture, moving/lifting heavy loads and static posture are the main physical factors retained in the regression models with odds ratios generally greater than 1.5 . This indicates that among all factors included in the regression models, these physical items have noticeable association with reported symptoms in different body regions. No psychological factor was retained in the regression models indicating that in this study population perceived psychological demands were not significantly associated with reported symptoms. Job tenure was the only demographic factor retained in the models that was a significant factor for the neck, elbows, wrists/hands, thighs and knees with OR ranging from 1.59 to 2.34 .

The association between the level of perceived demands and reported symptoms in different body regions is presented in Table 4. Since the number of nurses categorized as having low perceived physical demands was small, to perform meaningful statistical analysis the low and the medium levels of physical demands were combined to form a low-medium level of physical demands. As shown in Table 4, for all body regions the prevalence of symptoms was significantly higher in the group with a high level of physical demands $(P<.05)$ indicating an association between high perceived physical 
TABLE 3. Models Indicating Factors With the Strongest Influence on Musculoskeletal Disorders in the Nurses' Different Body Regions

\begin{tabular}{|c|c|c|c|c|}
\hline \multirow[b]{2}{*}{ Body Region } & \multicolumn{4}{|c|}{ Variables Retained in the Model } \\
\hline & Variables & OR & $95 \% \mathrm{Cl}$ & $P$-Value \\
\hline Neck & $\begin{array}{c}\text { Job tenure } \\
\text { Moving/lifting heavy loads } \\
\text { Head or arm awkward posture }\end{array}$ & $\begin{array}{l}2.12 \\
2.09 \\
2.20\end{array}$ & $\begin{array}{l}1.46-3.08 \\
1.15-3.80 \\
1.09-4.45\end{array}$ & $\begin{array}{l}<.0001 \\
.016 \\
.013\end{array}$ \\
\hline Shoulders & Body awkward posture & 2.01 & $1.20-3.38$ & .008 \\
\hline Elbows & $\begin{array}{c}\text { Job tenure } \\
\text { Static posture } \\
\text { Moving/lifting heavy loads }\end{array}$ & $\begin{array}{l}2.34 \\
2.15 \\
2.51\end{array}$ & $\begin{array}{l}1.47-3.71 \\
1.24-3.72 \\
1.10-5.69\end{array}$ & $\begin{array}{l}<.0001 \\
.006 \\
.028\end{array}$ \\
\hline Wrists/hands & $\begin{array}{c}\text { Job tenure } \\
\text { Apply pressure with hands/fingers } \\
\text { Intensive physical efforts }\end{array}$ & $\begin{array}{l}1.81 \\
2.38 \\
2.62\end{array}$ & $\begin{array}{l}1.21-2.70 \\
1.18-4.80 \\
1.02-6.73\end{array}$ & $\begin{array}{l}.004 \\
.016 \\
.045\end{array}$ \\
\hline Upper back & $\begin{array}{l}\text { Body awkward posture } \\
\text { Bent or twisted at waist }\end{array}$ & $\begin{array}{l}2.14 \\
1.74\end{array}$ & $\begin{array}{l}1.24-3.70 \\
1.06-2.85\end{array}$ & $\begin{array}{l}.006 \\
.028\end{array}$ \\
\hline Lower back & $\begin{array}{l}\text { Bent or twisted at waist } \\
\text { Body awkward posture }\end{array}$ & $\begin{array}{l}2.42 \\
1.17\end{array}$ & $\begin{array}{l}1.41-4.15 \\
1.25-3.75\end{array}$ & $\begin{array}{l}.001 \\
.006\end{array}$ \\
\hline Thighs & $\begin{array}{l}\text { Static posture } \\
\text { Job tenure }\end{array}$ & $\begin{array}{l}2.24 \\
1.57\end{array}$ & $\begin{array}{l}1.44-3.49 \\
1.06-2.33\end{array}$ & $\begin{array}{l}<.0001 \\
.025\end{array}$ \\
\hline Knees & $\begin{array}{l}\text { Job tenure } \\
\text { Static posture }\end{array}$ & $\begin{array}{l}1.59 \\
1.49\end{array}$ & $\begin{array}{l}1.12-4.08 \\
1.02-2.17\end{array}$ & $\begin{array}{l}.022 \\
.038\end{array}$ \\
\hline Legs/feet & $\begin{array}{l}\text { Bent or twisted at waist } \\
\text { Static posture }\end{array}$ & $\begin{array}{l}2.66 \\
1.56\end{array}$ & $\begin{array}{l}1.67-4.24 \\
1.18-2.30 \\
\end{array}$ & $\begin{array}{c}<.0001 \\
.034 \\
\end{array}$ \\
\hline
\end{tabular}

TABLE 4. Frequency of Reported Symptoms in the Nurses' Different Body Regions in the 12 months Prior to the Study $(n=641)$

\begin{tabular}{|c|c|c|c|c|c|c|}
\hline \multirow[b]{3}{*}{ Body Region } & \multicolumn{6}{|c|}{ Perceived Demands } \\
\hline & \multicolumn{3}{|c|}{ Physical } & \multicolumn{3}{|c|}{ Psychological } \\
\hline & Low-Medium ${ }^{a}(\%)$ & $\operatorname{High}^{\mathrm{b}}(\%)$ & $P$-value* & Low $^{c}(\%)$ & $\operatorname{High}^{\mathrm{d}}(\%)$ & $P$-value ${ }^{\star \star}$ \\
\hline Neck & 47 (26.9) & $173(40.5)$ & .0020 & 77 (33.9) & $143(37.7)$ & $>.050$ \\
\hline Shoulders & $56(31.5)$ & $193(43.9)$ & .0040 & $84(36.4)$ & 166 (42.3) & $>.050$ \\
\hline Elbows & $21(11.9)$ & $89(20.1)$ & .0150 & $41(18.1)$ & $71(17.9)$ & $>.050$ \\
\hline Wrists/hands & $54(30.5)$ & $190(43.1)$ & .0050 & $89(37.1)$ & $156(40.7)$ & $>.050$ \\
\hline Upper back & $76(42.7)$ & $212(48.6)$ & $>.0500$ & $106(46.3)$ & $183(46.9)$ & $>.050$ \\
\hline Lower back & $80(45.7)$ & $258(59.0)$ & .0030 & $121(53.5)$ & $219(56.0)$ & $>.050$ \\
\hline Thigh & 34 (19.5) & $146(33.8)$ & .0010 & $70(30.6)$ & $110(28.8)$ & $>.050$ \\
\hline Knees & $58(32.8)$ & $241(55.3)$ & $<.0001$ & $96(41.7)$ & $206(53.1)$ & .006 \\
\hline Legs/feet & $73(40.8)$ & $248(56.5)$ & .0010 & $117(48.3)$ & 207 (54.3) & $>.050$ \\
\hline
\end{tabular}

Notes. *-chi-square analysis of the prevalence of the symptoms between low-medium and high groups, ** - chi-square analysis of the prevalence of the symptoms between low and high groups; a-score 0-9, bscore 10-12, c-score 0-5, d-score 6-8.

demands and the prevalence rate of symptoms. For psychological demands, only the prevalence of reported symptoms in knees was significantly different between the low and the high psychological demands groups $(P<.05)$. 


\section{DISCUSSION}

This study has resulted in more detailed knowledge on the occurrence of MSDs in SUMS hospital nurses. Particularly significant results are discussed in the following sections.

\subsection{Prevalence of MSDs}

The questionnaire showed that MSDs were common among the nurses. A vast majority of the study population $(84.4 \%)$ had experienced some form of MSDs during the past 12 months. Lower back symptoms were found to be the most prevalent problem among the nurses studied with a past-year prevalence of $54.9 \%$. This is in accord with the findings of others $[12,15,16,17$, 19, 20]. A comparison of the results of this study with the results of a National Health Survey of Iran [28] revealed that the differences between the prevalence of MSDs among the nurses studied and the general Iranian population were significant (Table 5). This indicates that nursing can be considered as a high-risk occupation for developing MSDs. This is in full agreement with the findings of other studies $[4,11,12,13,14,15$, $16,17,18]$.

\subsection{Lost Working Days}

MSDs in the study population caused remarkable rate of sick leave in the past 12 months $(1,520$ days). With regards to the average daily working time of $8.3 \mathrm{hrs}$ (Table 1), this rate of sick leave is equal to 6.07 Full Time Equivalent (FTE), where FTE is considered to be 2080 yearly working hours of a person [29]. This shows that nearly $1 \%$ of the workforce who participated in this study were not productive and could not attend their jobs in the past year due to MSDs. Generalizing this feature to the overall nurses' population in the country would yield considerable lost working days during the preceding year. This estimation indicates that in this occupation, regardless of indirect costs and negative effects of MSDs on quality of services and productivity, MSDs have caused pronounced direct costs in the form of lost working days.

\subsection{Factors Associated With MSDs}

In general, statistical analysis of our data showed that perceived physical demands i.e., moving/ lifting heavy loads, awkward posture, static posture, applying pressure with hands/fingers, and intensive physical efforts were significantly associated with MSDs in different body regions (Table 3). Among the perceived physical demands investigated in this study, awkward posture was most frequently and strongly associated with reported MSDs in different body regions. This is in agreement with the findings of other researchers [13, 30]. Biomechanical studies have also showed the danger of awkward postures and other patient-handling activities that exceed body tolerances [12].

As no psychological item was retained in the regression models (Table 3), it could be inferred that psychological demands were not likely to be associated with reported symptoms. Of course, it is to be noted that Kerr et al. [31] found that when physical demands were included in a model of back problems, the significance of psychological demands disappeared. Statistical analysis also revealed that when comparing the low and the high perceived demands groups, only physical demands had influence on the prevalence rate of symptoms in almost all body regions with the exception of knees (Table 4). This indicates the consistency of the results of statistical analyses presented in Tables 3 and 4 .

TABLE 5. Comparison of Point Prevalence of Musculoskeletal Disorders in Neck, Back and Large Joints in General Iranian Population (Age: 15-69) and in the Study Population (Age: 22-66)

\begin{tabular}{lccc}
\hline Body Region & $\begin{array}{c}\text { Study Population } \\
\text { (\%) }\end{array}$ & $\begin{array}{c}\text { General Iranian Population } \\
\text { (\%) }\end{array}$ & P-value* $^{*}$ \\
\hline Neck & 22.40 & 10.20 & $<.0001$ \\
Upper and lower back & 49.30 & 25.29 & $<.0001$ \\
Large joints $^{\text {a }}$ & 60.40 & 20.00 & $<.0001$ \\
\hline
\end{tabular}

Notes. *-test of proportion; a-including shoulders, elbows, wrists, knees and ankles. 


\subsection{Reliability Tests}

The results of a reliability test revealed that for almost all subjective variables the Spearman correlation coefficient were greater than .60 , which could be considered acceptable for this kind of variables. McNemar and Wilcoxon tests also showed no significant difference between responses for the two occasions. The Pearson correlation coefficient for quantitative variables was greater than .9 indicating acceptable reliability of the administered questionnaire.

\section{CONCLUSION}

There was a high rate of MSDs among the nurses studied. The results of this study showed the importance of perceived physical demands in relation to reported MSDs in different body regions. No association was found between perceived psychological demands and reported symptoms. Among the perceived physical demands studied here, those involving awkward posture were most frequently and strongly associated with reported MSDs in nearly all body regions. Based on the findings of this study, it can be concluded that any intervention program for preventing or reducing MSDs among SUMS hospital nurses should focus on reducing physical demands, particularly excessive postural demands.

\section{REFERENCES}

1. Shahnavaz H. Workplace injuries in the developing countries. Ergonomics. 1987;30(2):397-404.

2. Genaidy AM, Al-Shedi AA, Shell RL. Ergonomics risk assessment: preliminary guidelines for analysis of repetition, force and posture. J Hum Ergol (Tokyo). 1993;22:45-55.

3. Kemmlert K. Labor inspectorate investigation for the prevention of occupational musculo-skeletal injuries [licentiate thesis]. Solna, Sweden: National Institute of Occupational Health; 1994.

4. Smith DR, Sato M, Miyajima T, Mizutani T, Yamagata Z. Musculoskeletal disorders self-reported by female nursing students in central Japan: a complete cross-sectional survey. Int $\mathrm{J}$ Nurs Stud. 2003;40:725-9.

5. Maul A, Laubli T, Klipstein A, Krueger H. Course of low back pain among nurses: a longitudinal study across eight years. Occup Environ Med. 2003:60:497-503.

6. Menzel NN. Back pain prevalence in nursing personnel: measurement issues. AAOHN J. 2004;52(2):54-65.

7. Bernard B, editor. Musculoskeletal disorders and workplace factors: a critical review of epidemiologic evidence for work-related musculoskeletal disorders of the neck, upper extremity, and low back (DHHS/NIOSH publication No. 97-141). Washington, DC, USA: U.S. Department of Health and Human Services (DHHS); 1997.

8. Linton SJ, Kamwendo K. Risk factors in the psychosocial work environment for neck and shoulder pain in secretaries. J Occup Med. 1989;31(7):609-13.

9. Carter JB, Banister EW. Musculoskeletal problems in VDT work: a review. Ergonomics. 1994;37(10):1623-48.

10. Weiser S. Psychosocial aspects of occupational musculoskeletal disorders. In: Nordin M, Andersson GBJ, Pope MH, editors. Musculoskeletal disorders in the workplace: principles and practice. St. Louis, MO, USA: Mosby-Year Book; 1997.

11. Engkvist IL, Hagberg M, Wigaeus-Hjelm E. Interview protocols and ergonomics checklist for analyzing overexertion back accidents among nursing personnel. Appl Ergon. 1995;26(3):213-20.

12. Hignett $S$. Postural analysis of nursing work. Appl Ergon. 1996;27(3):171-6.

13. Trinkoff AM, Lipscomb JA, Geiger-Brown J, Storr CL, Brady BA. Perceived physical demands and reported musculoskeletal problems in registered nurses. Am J Prev Med. 2003;24(30):270-5.

14. Menzel NN, Brooks SM, Bernard TE, Nelson A. The physical workload of nursing personnel: association with musculoskeletal discomfort. Int J Nurs Stud. 2004;41:859-67.

15. Larese F, Fiorito A. Musculoskeletal disorders in hospital nurses: a comparison 
between two hospitals. Ergonomics. 1994;37:1205-11.

16. Smedley J, Egger P, Cooper C, Coggon D. Manual handling activities and risk of low back pain in nurses. Occup Environ Med. 1995;52:160-3.

17. Engels JA, van der Gulden JW, Senden TF, van't Hof B. Work related risk factors for musculoskeletal complaints in the nursing profession: results of a questionnaire survey. Occup Environ Med. 1996;53:636-41.

18. Ando S, Yuichiro O, Shimaoka M, Hattori S, Hori F, Takeuchi Y. Associations of selfestimated workloads with musculoskeletal symptoms among hospital nurses. Occup Environ Med. 2000;57:211-6.

19. Hignett $S$, Richardson B. Manual handling human loads in a hospital: an exploratory study to identify nurses' perceptions. Appl Ergon. 1995;26(3):221-6.

20. Botha WE, Bridger RS. Anthropometric variability, equipment usability and musculoskeletal pain in a group of nurses in the Western Cape. Appl Ergon. 1998;29(6):481-90.

21. Owen BD. Preventing injuries using an ergonomic approach. AORN J. 2000; 72:1031-6.

22. Hignett S. Using computerized OWAS for postural analysis of nursing work. In: Robertson SA, editor. Contemporary ergonomics. Proceedings of the Ergonomics Society's 1994 Annual Conference. London, UK: Taylor \& Francis; 1994. p. 253-8.

23. Engels JA, Landeweerd JA, Kant Y. An OWAS-based analysis of nurses' working postures. Ergonomics. 1994;37(5):909-19.

24. Kuorinka I, Jonsson B, Kilbom A, Vinterberg H, Biering-Sorensen F,
Andersson G, Jorgensen K. Standardised Nordic questionnaires for the analysis of musculoskeletal symptoms. Appl Ergon. 1987;18(3):233-7.

25. Karasek RA. Job Content Questionnaire and user's guide. Lowell, MA, USA: Department of Work Environment, University of Massachusetts; 1985.

26. Karasek RA, Brisson C, Kawakami N, Houtman I, Bongers P, Amick B. The Job Content Questionnaire (JCQ): an instrument for internationally comparative assessments of psychosocial characteristics. J Occup Health Psychol. 1998;3(4):322-55.

27. Colin D. Beginning research in psychology: a practical guide to research methods and statistics. Oxford, UK: Blackwell; 1995.

28. National Research Center of Medical Sciences of Iran. National health survey of Iran: overall country. Tehran, Iran: Health Ministry of I.R. Iran; 2001. In Persian.

29. U.S. Department of Energy. Laboratory operations board. Third report of the external members to the Secretary of Energy Advisory Board (September 1997). Attachment II: Laboratory productivity metrics. Appendix 1 (April 1996): definitions and instructions. Retrieved September 15, 2006 from: http://www.seab .energy.gov/sub/metrics.html

30. Punnett L, Fine LJ, Keyserling WM, Herrin GD, Chaffin DB. Shoulder disorders and postural stress in automobile assembly work. Scand J Work Environ Health. 2000;26:283-91.

31. Kerr MS, Frank JW, Shannon HS, Norman RW, Wells RP, Neumann WP, Bombardier C. Biomechanical and psychological risk factors for low back pain at work. Am J Public Health. 2001;91:1069-75. 


\section{Appendix}

\section{A1. Physical Demand Items}

My job requires

- lots of physical effort ${ }^{1}$

- rapid and continuous physical activity ${ }^{1}$

In my job, I

- often move/lift very heavy loads ${ }^{1}$

- work for long periods with my head or arms in awkward positions ${ }^{1}$

- work for long periods with my body in awkward positions ${ }^{1}$

How often in a typical workday do you

- lift or lower patients/objects to/from floor ${ }^{2}$

- lift or lower objects to/from shoulder height ${ }^{2}$

- work while bent or twisted at waist ${ }^{2}$

- push/pull heavy objects or people ${ }^{2}$

- stand in one place/static position $(>30 \mathrm{~min})^{2}$

- perform repetitive motions with hands/wrists ${ }^{2}$

- apply pressure with hands/fingers (e.g., to prevent bleeding) ${ }^{2}$

\section{A2. Psychological Demand Items ${ }^{1}$}

My job requires

- working very hard

- working very fast

- an excessive amount of work

- long periods of intense concentration on the task

- enough time to get the job done

My job

- is free from conflicting demands that others make

- has tasks that are often interrupted before they can be completed

- requires waiting for work from other people or departments

\footnotetext{
1 original item from the Job Content Questionnaire [25]

item from Trinkoff, Lipscomb, Geiger-Brown, et al. [13]
} 